\title{
INTERBAND ABSORPTION IN InGaAs/GaAs QUANTUM WELL AT HIGH HYDROSTATIC PRESSURE
}

\author{
T.P. Sosin*, P. Perlin, W. Trzeciakowski, \\ High Pressure Research Center, Polish Academy of Sciences \\ Sokołowska 29, 01-142 Warszawa, Poland \\ AND R. TOBER \\ U.S. Army Laboratory Command \\ 2800 Powder Mill Road, Adelphi, MD 20783, USA
}

\begin{abstract}
The interband absorption of strained InGaAs/GaAs multiple quantum well was studied at room temperature for pressures up to $5.5 \mathrm{GPa}$. Three absorption lines were attributed to the excitonic transitions hh1-e1, lh1-e1 and $\mathrm{hh} 2-\mathrm{e} 2$. They were visible until pressure of about $5 \mathrm{GPa}$ which is above the $\Gamma-X$ crossover for this system. Pressure coefficients of the observed lines were compared with the literature data. The origin of broadening of the lines above $\Gamma-X$ crossover is discussed.
\end{abstract}

PACS numbers: 73.20.Dx

\section{Introduction}

InGaAs based quantum wells ( $Q W$ ) have been extensively studied because of their potential applications in telecommunications. In contrast to GaAs/GaAlAs structures there is a substantial lattice mismatch between the InGaAs well and GaAs barrier. This leads to the biaxial strain in InGaAs layers which causes large splitting of the heavy and light-holes states. For this reason the hydrostatic and uniaxial deformation potentials are crucial for the determination of the electronic states of this system.

The interband absorption of InGaAs/GaAs QW contains distinct excitonic lines even at room temperature. These lines occur below the GaAs substrate absorption edge and therefore the QW absorption can be measured without the necessity of etching the substrate away. This allows for the study of the ground and excited states of the system which is an advantage over photoluminescence experiments [1-3].

*Also at the Institute of Physics, Warsaw University of Technology, Koszykowa 75, 00-662 Warszawa, Poland. 


\section{Samples}

Our samples were grown by molecular beam epitaxy (MBE) on Si-doped ( $n=$ $10^{18} \mathrm{~cm}^{-3}$ ) GaAs substrate. After a buffer layer of undoped GaAs the multiple QW was grown with 50 repetitions of $\operatorname{In}_{0.14} \mathrm{Ga}_{0.86}$ As wells (nominal width $104 \AA$ ) and GaAs barriers (nominal width $200 \AA$ ). Finally, a $1000 \AA$ layer of $p^{+}$(Be doped) GaAs cap was grown. Thus, the multiple $Q W$ structure is in the insulating region of a $n-i-p$ diode and the wells are subject to a weak electric field of a few $\mathrm{kV} / \mathrm{cm}$. This field is too weak to cause appreciable shifts of the lowest levels but it can lead to the appearance of some forbidden transitions.

For high-pressure experiments in the diamond anvil cell the substrate was thinned down to $40 \mu \mathrm{m}$.

\section{Experimental}

Diamond anvil cell was used to generate pressure up to $6 \mathrm{GPa}$. Methanolethanol mixture was used as a pressure transmitting medium. Pressure was measured by determining the position of absorption edge of the substrate (GaAs) using the equation: $E_{\mathrm{g}}=E_{\mathrm{g}}(0)+116 p-3 p^{2}$ [4]. Here $p$ is expressed in GPa and energy in meV. This method is much more accurate than the calibration with the ruby gauge and works even above the $\Gamma-X$ crossover in GaAs.

The light from a halogen lamp was passed through a $100 \mu \mathrm{m}$ pinhole and was focused on the sample. Transmitted light was analysed by Spex 500M monochromator with cooled GaAs photomultiplier and photon counting system. Absorption (optical density) was determined from the logarithm of the ratio of intensity of light passing through the sample and by the sample.

\section{Results}

We observe three absorption lines in the spectrum of the sample. The line denoted $A$ is the transition from the first heavy-hole state to the first electron state, i.e. hh1-e1. Line $B$ is attributed to the similar transition from the light-hole state (lh1-e1) and line $C$ to the transition hh2-e2. This tentative assignment is based on the literature data for similar samples $[5,6]$.

In Fig. 1 we show the absorption spectra at various pressures. Up to about $4 \mathrm{GPa}$ absorption spectra are blue shifted without any noticeable change in their shape. Between 4.8 and $5.2 \mathrm{GPa}$ absorption lines abruptly disappear and we observe only smooth absorption spectrum dominated by the substrate absorption edge. We followed the position of all three states (see Fig. 2) and we determined the pressure coefficient of the ground state which can be described by equation: $E_{\mathrm{g}}=E_{\mathrm{g}}(0)+112 p-2 p^{2}$ which is very close to the value characteristic of bulk GaAs gap. We determine the pressure coefficient of the higher states by plotting the energy separation of the $B$ and $C$ peaks from the $A$ peak at each pressure (Fig. 3).

We can observe that the higher levels have slightly higher pressure coefficients, it means that linear coefficient for $B$ is $114 \mathrm{meV} / \mathrm{GPa}$ and for $C 117 \mathrm{meV} / \mathrm{GPa}$. 


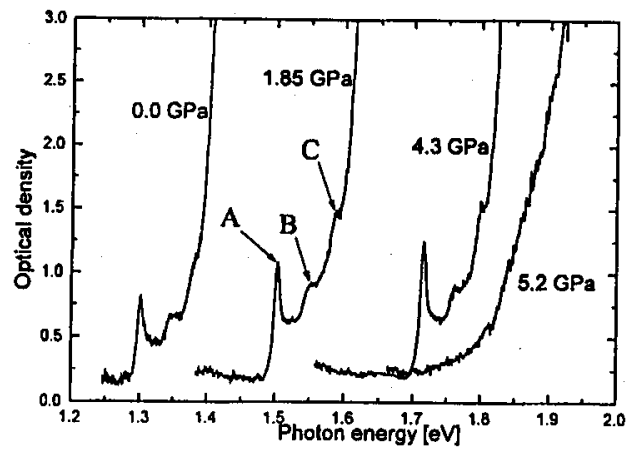

Fig. 1. Absorption spectra of GaInAs quantum well at various pressures, measured at room temperature.
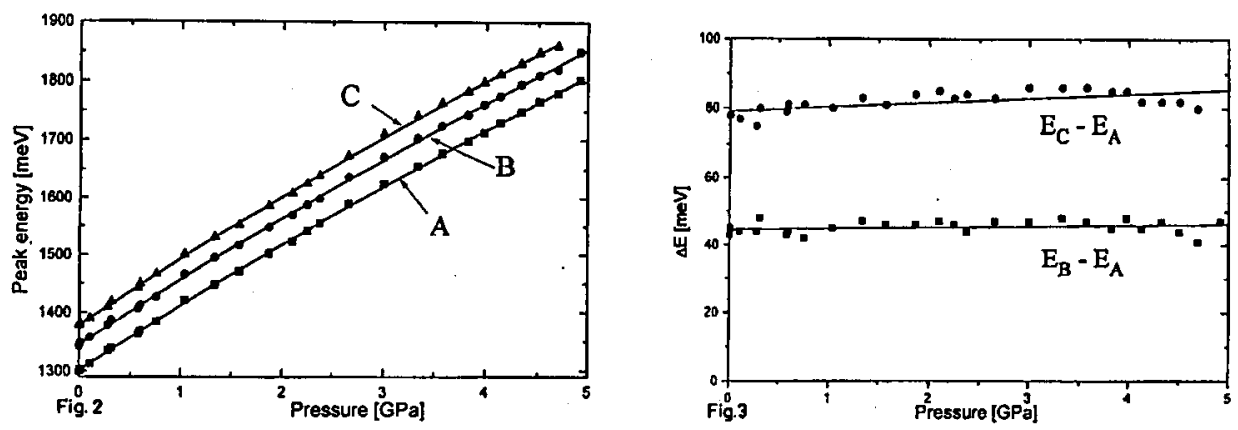

Fig. 2. Pressure dependence of the positions of $A, B, C$ absorption peaks.

Fig. 3 Energy separation between $B$ and $A$ transition and $C$ and $A$ transition.

\section{Discussion}

We would like to point out the following results:

1. Pressure coefficient of line $A(112 \mathrm{meV} / \mathrm{GPa})$ is very close to that of bulk GaAs (116 meV/GPa). This is in excellent agreement with results of Ref. [7] but substantially higher than the values reported in Refs. [1] and [3]. This is partly due to the fact that the data in Refs. [1] and [3] were fitted with a straight line while we clearly see a sublinear behaviour, similarly to Ref. [7]. Our values seem to contradict the strong indium-composition dependence of the pressure coefficient proposed in Ref. [1]. It has to be stressed, though, that our results (as well as those of Ref. [7]) were obtained at $300 \mathrm{~K}$ while the luminescence data from Refs. [1] and [3] were obtained at $80 \mathrm{~K}$ and at $20 \mathrm{~K}$.

2. Small increase in the distance between the ground and excited state can be explained by increase in the barrier heights with pressure, in agreement with the findings of Ref. [3].

3. Disappearance of the excitonic absorption above the crossover pressure 
has a different origin than the disappearance of the luminescence signal. The latter occurs mainly because of the depopulation of the $\Gamma$ levels while the former is due to the resonant Fano effect. Above the crossover pressure the $\Gamma$ levels become degenerate with the $X$ continuum. In GaAs quantum wells the $X$ continuum is lower in the barriers while in InGaAs wells the lowest $X$ states seem to be in the well layers (see Ref. [8]). The mixing of $\Gamma$ and $X$ states which leads to the broadening of the $\Gamma$ absorption line occurs mainly due to the interaction with zone-edge optical phonons $[9,10]$. In the case of GaAs wells the mixing is mainly due to the presence of the interface which destroys the translational symmetry along the growth direction [4]. The temperature dependence of these two mixing mechanisms should be very different and we plan to investigate this point by performing low-temperature measurements. The $\Gamma-X$ mixing through phonons and through the interface is very important in the double-barrier resonant tunnelling structures which are based on GaAs or on InGaAs quantum wells.

\section{Acknowledgment}

We gratefully acknowledge financial support by the Committee for Scientific Research - grant number 773369203.

\section{References}

[1] V.A. Wilkinson, A.D. Prins, J.D. Lambkin, E.P. O'Reilly, D.J. Dunstan, L.K. Howard, Phys. Rev. B 42, 3113 (1990).

[2] S.G. Lyapin, M.I. Eremets, O.A. Krasnovskii, A.M. Shirokov, V.D. Kulakovskii, T.G. Andersson, Z.G. Chen, in: Proc. IV Int. Conference on High Pressure in Semiconductor Physics, Porto Carras 1990, Eds. D.S. Kyriakos, O.E. Valassides, Aristotle University of Thessaloniki, Thessaloniki 1990.

[3] G.H. Li, B.Z. Zheng, H.X. Han, Z.P. Wang, T.G. Andersson, Z.G. Chen, Phys. Rev. B 45, 3489 (1992).

[4] P. Perlin, W. Trzeciakowski, E. Litwin-Staszewska, J. Muszalski, M. Micovic, unpublished.

[5] G. Ji, D. Huang, U.K. Reddy, T.S. Henderson, R. Houdré, H. Morkoc, J. Appl. Phys. 62, 3366 (1987).

[6] C. Shwe, M. Gal, Appl. Phys. Lett. 57, 1910 (1990).

[7] W. Shan, X.M. Fang, D. Li, S. Jiang, S.C. Shen, H.Q. Hou, W. Feng, J.M. Zhou, Appl. Phys. Lett. 57, 475 (1990).

[8] V.A. Wilkinson, A.D. Prins, D.J. Dunstan, L.K. Howard, M.T. Emeny, J. Electron. Mater. 20, 509 (1991).

[9] A.R. Goñi, A. Cantarero, K. Syassen, M. Cardona, Phys. Rev. B 41, 10111 (1990).

[10] S. Satpathy, M. Chandrasekhar, H.R. Chandrasekhar, U. Venkateswaran, Phys. Rev. B 44, 11339 (1991). 